\title{
REFLEXIONES SOBRE LA
}

\section{EDUCACIÓN CARCELARIA.}

EXPERIENCIAEN UNESTABLECIMIENTO

PENITENCIARIO COLOMBIANO

\section{REFLECTIONS ON PRISON EDUCATION. EXPERIENCE INACOLOMBIAN PRISON}

\section{Héctor Fabián Cristancho Guzmán ${ }^{1}$}

Universidad Pedagógica y Tecnológica de

Colombia, Duitama, Boyacá, Colombia

\section{RESUMEN}

Las personas privadas de la libertad (PPL) rechazan los programas educativos, lo que resulta en una resocialización ineficiente. En varios países como España, Argentina, Brasil, Colombia, Costa Rica, El Salvador y México, se han realizado investigaciones e implementado programas con la finalidad contribuir en la formación de las PPL. Se ha comprobado que no todas las intervenciones resultan ser efectivas, ya que los efectos positivos se mantienen mientas se aplica el programa y no perduran en el tiempo.

$1 \quad$ Licenciado en Educación Industrial. Estudiante de Maestría en TIC Aplicadas a las Ciencias de la Educación, docente de pregrado de la Universidad Pedagógica y Tecnológica de Colombia sede Duitama. hector.cristancho@ uptc.edu.co https://orcid.org/0000-0002-9670-3522
Se plantea que esta situación se debe a que no se ha considerado la problemática y necesidades reales de la comunidad de estudio y se aplican dichos programas de una forma 'empírica'. El objetivo del artículo es presentar unas reflexiones acerca del tratamiento penitenciario a partir de una experiencia de investigación en un establecimiento penitenciario colombiano.

PALABRAS CLAVE: tratamiento penitenciario, resocialización, educación carcelaria.

\section{ABSTRACT}

People deprived of liberty (PPL) reject educational programs, resulting in inefficient resocialization. In several countries such as Spain, Argentina, Brazil, Colombia, Costa Rica, El Salvador and Mexico, research has been carried out and programs have been implemented with 
the aim of training the PPL. It has been proven that not all interventions are effective, since the positive effects are maintained while the program is applied and do not last over time.

It is argued that this situation is due to the fact that the real problems and needs of the study community have not been considered and these programs are applied in an "empirical" way. The aim of the article is to present some reflections on prison treatment based on a research experience in a prison.

KEYWORDS: prison treatment, resocialization, prison education.

A partir del desarrollo de un estudio con personas privadas de la libertad, y desde la perspectiva de la pedagogía social, una de las ramas de la pedagogía que se orienta a la educación de los menos favorecidos, que también han sido llamados marginados sociales 'término que ha tenido mucha controversia,' se presentan las siguientes reflexiones que podrían contribuir, prevenir o coayudar en la intervención socioeducativa con los privados de la libertad partiendo de la identificación de las necesidades sociales y educativas de cada establecimiento considerándolo como un entorno individual y particular

Para empezar, Fernandes (2008) entrega cifras alarmantes de un centro penitenciario en Brasil, las cuales son semejantes en la mayoria de centros penitenciarios de latinoamérica; todos los condenados tienen el derecho y la posibilidad de acceder a programas educativos, pero apenas el $17,3 \%$ de ellos participan en actividades educativas. El problema no radica en la no disposicipon de la población para participar, lo que sucede es que no se dan la condiciones apropiadas para ello debido a la forma en que tienen organizados ealguos establecimientos en términos de metodología, horarios y distribución del entorno físico.
En el tratamiento penitenciario, conocer la realidad educativa de una comunidad resulta de gran importancia, pues de allí recae la efectividad de las intervenciones realizadas. De acuerdo con Molano (2011) es importante garantizar la culminación de los estudios de las PPL en los diferentes niveles educativos. El mismo autor manifiesta que, aunque la norma brinda todas las garantías, la realidad es diferente, es decir que se están vulnerando los derechos de los privados de libertad Huertas (2015), y estas apreciaciones se comprobaron con el desarrollo de esta investigación.

Una dimensión que aparece como dominante es la red social, la cual está compuesta por una red social interna que corresponde a los privados de libertad y otra corresponde a la red social externa, que corresponde a la sociedad en general, ya que, de acuerdo a las apreciaciones de privados de la libertad, la red social externa influye directamente sobre ellos debido a la marginación y exclusión al quedar en libertad. Esta apreciación está en consonancia con Blazich (2007), quien establece que las condiciones del entorno de los privados de libertad y ecosistema en general hace que su población sea vulnerable, susceptible a la exclusión, a ser marginados y a la violencia.

En cuanto a la red social interna, está dispuesta a resocializarse y capacitarse, pero el mismo entorno no lo permite ya que, como establece Marcuello et al. (2011) quienes encuentran que se termina por imponer una consolidacion de identidades prisionizadas a modo de desocialización, les toca adaptarse a las reglas que imponen entre PPL y se ven obligados a adoptar conductas que van en contra de la ley para que su estancia sea más llevadera. Esto también has sido probado por la autora Rúa (2015) quien encontró que en estos entornos se conforman estructuras sociales, económicas y políticas. Por la misma razón debe tenerse en consideración los estudios de Fanny T. Añaños (2013), 
Concepción Yagüe Olmos (2013), William Frank Español Sierra (2014), Bethy Edith Moreno Farías (2014), quienes han demostrado que el hecho de privar de la libertad a estas personas no es suficiente para su resocialización. De la misma manera, ha de considerarse a todo el personal de los establecimientos penitenciarios, ya que la responsabilidad no recae únicamente en los docentes encargados de los programas, influye el personal encargado de la seguridad, ya que, según los privados de libertad, en muchas ocasiones son agresivos con ellos y esto agrava la convivencia. Por el contrario, desean que el personal de vigilancia los comprenda y los trate como seres humanos y respeten sus derechos.

En cuanto a la red social externa, es una población que también debe ser concientizada para la convivencia y reconciliación. De la misma manera los investigadores Gomez, Rodriguez y \& Viloria (2011) establecen que se deben considerar tanto factores psicológicos como factores sociales para poder comprender algunas conductas criminales. El mismo autor y Abaunza et al. (2016) resaltan la importancia de considear aspectos familiares y laborales, pues resultan ser los factores psicosociales más influyentes en las conductas delictivas. Lo anterior se corrobora con las apreciaciones de los privados de libertad de este estudio, pues consideran que la falta de educación no es precisamente la razón para cometer delitos, sino que influye directamente la sociedad al marginarlos, excluirlos y negarles oportunidades.

Solbes (2006), manifiesta que la población de privados de la libertad son personas inseguras, que no son capaces de afrontar el fracaso y por tanto la desconfianza al enfrentarse a nuevas experiencias. En contra a esta apreciación, los privados de libertad están dispuestos a superarse y reconciliarse con la sociedad. Durante la investigación se mostraron muy animados ya que, según ellos, estaban aprendiendo algo útil y diferente a las clases monótonas del establecimiento. Entonces se puede establecer que estas personas se desmotivan, no por el hecho de estudiar sino por las características del programa ofrecido.

Por otra parte, Paba (2016) demuestra que las reclusas tienen una autopercepcion muy positivista ya que ellas consideran que son buenas personas, con buenos valores y aptas para vivir en sociedad. Lo preocupante de este estudio es que asi mismo, las reclusas manifiestan no haber tenido ningún cambio positivo durante su estancia en prision. Esta misma apreciación la comparten los privados de libertad que participaron de este estudio, ya que consideran que las clases que toman en el establecimiento no les sirve para nada y por tanto no se genera un cambio positivo en ellos.

Molano (2011) resalta que los privados de libertad viven en precarias condiciones y que esta situación se agrava con la sobrepoblación y falta de recursos. Esta situación se observó durante el estudio ya que las instalaciones educativas se constituyen en un entorno con restricciones y limitaciones en cuanto a recursos educativos como libros, computadores, entre otros. Los privados de la libertad desean poder acceder libros a su antojo, es decir, con temas de su interés, pero no en todos los establecimientos penitenciarios se les permite. Gelana (2017) destaca que hay establecimientos en los cuales se permite a los privados de libertad a que lean de acuerdo a sus necesidades con el fin de promover su capacitación.

Rojas (2003) postula la necesidad de considerar e integrar la virtualización de los procesos educativos. El autor encuentra que, al formar a los internos a distancia, se fomenta la autonomía a pesar de las restricciones que impone el establecimiento penitenciario. El acceso a los computadores también es un deseo de los privados de libertad, pues consideran que en la actualidad necesitan tener conocimientos ofimáticos para desempeñarse en diferentes 
áreas. Lo anterior debe considerarse con urgencia para reducir brechas digitales y asi atender las necesidades de la población de privados de la libertad Molano (2011); Barreiro, et al. (2013). Por lo anterior se encontró que, a consideración de los privados de la libertad, al quedar en libertad tenían problemas de adaptación al continuar sus estudios por fuera del establecimiento debido a las falencias y restricciones del tratamiento ofrecido por algunos establecimientos.

Por otra parte Scarfó (2006) resalta la importancia de que las cárceles muestren una imagen diferente de sus entorno físico, es decir, que aunque es evidente que son espacios con necesidades y condiciones inapropiadas, debe ofrecer al interno y a sus trabajadores una visión de posibilidades para la superación.

Toro y Valencia (2005) establecen que educar en prisiones es un reto debido a las razones expuestas y además las condiciones de salubridad. Lo anterior se pudo constatar debido a la presencia de plagas que afectan la salud de los privados de la libertad y que se traducen en distracciones durante las capacitaciones, lo que las hace inproductivas.

Por otra parte, Aysha Kerr (2018) manifiesta que el uso de TIC ha permitido a la sociedad acercarse a bastas cantidades de información y de personas permitiendo un cambio positivo en lo personal y profesional. Pero estos beneficios no han llegado a toda la población en general, por ejemplo, las cárceles. Así mismo, Gutiérrez, Viedma, y Callejo (2010) señalan la importancia de innovar en el tratamiento que se da a esta población, como por ejemplo, incluir tecnologías de información y comunicación para llevar a cavo estudios de educación superior, lo cual resultaría positivo para la mayoria de los participantes ya que según los mismos internos, ayuda a que el tiempo en prision sea mas llevadero, y ayuda a sentirse mejor con si mismos.
Además, es necesario considerar las características de la población de privados de libertad en cuanto a conocimientos previos, ya que muchos de ellos se encuentran inscritos en programas muy atrasados respecto a su nivel de estudios y esto ocasiona que se desmotiven o que pierdan el tiempo asistiendo a clases que no les aporta nada en su resocialización. Diez (2009) encuentra que es importante tener en cuenta las experiencias de vida de las personas adultas, ya que todas las participantes de su investigación, utilizaban las matemáticas en sus quehaceres diarios sin ser conscientes de ello, por ejemplo, para contar el dinero del mercado, hacer restas al pagar servicios, entre otros. Además, encontraron que el hecho de aprender matemáticas formalmente, aumentó su autoestima y motivación. De la misma manera sucedió en este estudio, ya que mientras se implementaba un programa en matemáticas, encontraron la utilidad en las mismas, aprendieron nuevos conceptos y se mostraron siempre motivados.

Salamanca (2016); Añaños et al. (2012); Añaños y Yagüe (2013) y Hernández (2017) consideran que para garantizar la participacion efectiva de las PPL en programas educativos se debe considerar que éstos deben ser de calidad, ya que no basta con ofrecer programas y tener personal para ello si no están adecuados para las circunstancias. Además, Gelana (2017), postula la necesidad de capacitaciones basadas en metodologías activas y participativas.

Scarfó, (2006) establece cuatro características que deben tener los programas educativos en los centros penitenciarios: accesibilidad,sin restricciones; asequibilidad, diversidad de programas educativos; adaptabilidad, programas adecuados para la población; y aceptabilidad y que los métodos utilizados estén acorde a los derechos humanos. Lo anterior resulta prometedor pero la realidad de muchos establecimientos es que los programas ofrecidos 
no cuentan con la mayoría de las características que propone el autor, lo cual enciende las alarmas e invita a la comunidad educativa a intervenir con esta población.

Finalmente, Crabbe (2016) resalta la importancia de que exista una cultura de aprendizaje en las cárceles, tanto en las celdas como en las aulas de clase. Además, estas clases deben ser estructuradas y secuenciales las cuales permitan seguir a los privados de libertad, aun cuando queden en libertad. Es una realidad que tampoco se evidencia cumplimiento o concordancia con lo que propone el autor, en algunos establecimientos penitenciarios, ya que las clases parecen no tener un objetivo bien definido y se les restringe recursos, como los libros, los cuales podrían llevar a sus celdas y aprovechar el tiempo de encierro. Lo último resultaría en una buena estrategia para mitigar los efectos negativos que se estimulan en el tiempo de ocio Farley y Pike (2016).

Para concluir, Los autores Aishah y Zeema (2017), Del Pozo, Jiménez, y Turbi (2013) y Del Pozo y Añaños (2012), Añaños y Yagüe (2013), Ayuso (2001), Brasil (2008), Castillo y Ruiz (2007) resaltan en sus trabajos que, la participación de las personas privadas de la libertad en programas de capacitación, contribuye a su resocialización; lo cual concuerda con lo encontrado en esta investigación, como lo manifestaron de los privados de libertad, que necesitan más programas de capacitación pero orientados al desarrollo de habilidades laborales, para ellos, las clases de primaria no aportan lo suficiente para lograr una reinserción adecuada a la sociedad.

Por otra parte, los autores Abaunza, Mendoza, Paredes, y Bustos (2016), Añaños et al. (2013), Fernandes (2008) Marcuello et al. (2011) manifiestan que fenómeno mundial es que las conductas delictivas de las PPL se mantienen y refuerzan al interior del centro penitenciario debido a que hay poca participación en programas educativos. En efecto, con los resultados de este estudio se comprueba que esta apreciación es verdadera y una realidad actual ya que los privados de libertad lo manifestaron. Esto implica a que la comunidad educativa en general realice intervenciones constantes de capacitación a los privados de libertad.

Es de destacar como cada espacio carcelario en diferentes países puede asumir características conductuales diferentes. Este estudio solo da cuenta de un país latinoamericano, con muchas carencias donde en amiente se destaca por la inconformidad y la desconfianza.

Se consideran, estas reflexiones, como una oportunidad de mejoramiento de la intervención con personas privadas de la libertad y además concientizar en que debemos hacerles sentir reconocidos como personas y con ello, sus disposiciones, intereses y valores.

\section{REFERENCIAS BIBLIOGRÁFICAS}

Fernandes Julião, E. (2008). La Educación De Jóvenes $Y$ Adultos En Situacion De Privación De Libertad: Desafíos Y Perspectivas Para La Consolidación De Una Política Nacional. En Unesco, Educación En Prisiones De Latinoamérica (Págs. 65-76). Brasilia

Molano Gómez, E. A. (2011). La Educación Como Medio Para La Rehabilitación,Resocialización Y Redención De Pena Del Interno En Los Establecimientos Penitenciarios $Y$ Carcelarios De Colombia Establecimientos Penitenciarios $Y$ Carcelarios De Colombia. Bogotá.

Huertas Díaz, O. (2015). Sistema Penal Y Hacinamiento Carcelario. Análisis Al Estado De Cosas Inconstitucionales En Las Prisiones Colombianas. Revista Jurídica Derecho, 15-24.

Blazich, G. S. (2007). La Educación En Contextos De Encierro. Revista Iberoamericana De Deducación, 53-60. 
Marcuello Servós, C., \& García Martínez, J. (2011). La Cárcel Como Espacio De DeSocialización Ciudadana: ¿Fracaso Del Sistema Español? Portularia, 49-60.

Rúa, L. M. (2015). Construcciones Socioespaciales En El Encierro: La Cárcel Bellavista. Revista Colombiana De Geografía, 171-194.

Añaños Bedriñana, F., \& Yagüe Olmos, C. (2013). Educación Social En Prisiones. Planteamientos Iniciales $Y$ Políticas Encaminadas Hacia L Areinsercion Desde La Perspectiva De Género. Pedagogía Social, 7-12.

Español Sierra, W., \& Moreno Farías, B. (2014). La Educación En Cárceles,Una Experiencia Desde Un Establecimiento De Reclusión. Hojas Y Hablas, 7-19.

Gomez Rios , A. M., Rodriguez Urriago, N. R., \& Viloria Acero, S. C. (2011). Caracterización Psicosocial De Peronas Privadas De La Libertad Carcel Distrital De Bogotá. Bogotá.

Abaunza Forero, C. I., Mendoza Molina, M., Paredes Álvarez, G., \& Bustos Benítez, P. (2016). Familia Y Privación De La Libertad En Colombia. Bogotá: Universidad Del Rosario.

Martín Solbes, V. M. (2006). Actitudes De Los Internados En Prision, Menores De Veintiun Años, Ante La Funcion Reeducadora Del Medio Penitenciario En El Ambito Andaluz. Andaluz.

Paba Barbosa, C., Ortega Pacheco, Y. J., Campillo Gómez, M. A., Hernández Bolívar, M. F., Martínez Rudas, M., \& Porto Corredor, D. M. (2016). Autopercepción De Las Reclusas Reincidentes En Una Cárcel Colombiana Y Su Relación Con El Crimen. Collectivus, Revista De Ciencias Sociales, 67-86.

Viedma Rojas, A. (2003). La Educación A Distancia En Prisión. Estudio De Los Alumnos De La Uned Internos En Centros Penitenciarios.
Revista Iberoamericana De Educación A Distancia, 97-120.

Gelana Kabeta, G. (2017). Assessing The Practices Of Prison Education In Selected Prisons Of Amhara National Regional State, Ethiopia. Etiopía.

Barreiro Gen, M., Novo Corti, M. I., \& Candamio, L. V. (2013). E-Prisons And New Technologies: Ict As A Mechanism Of Social Inclusion Of Prisoners. International Journal Of Knowledge Society Research, 1-10.

Scarfó, F. J. (2006). Los Finales De La Educación Básica En Las Cárceles De La Provincia De Buenos Aires. Buenos Aires.

Toro Valencia, B. N. (2005). Educación Superior En Las Carceles Colombianas.

Gutiérrez Brito, J., Viedma Rojas, A., \& Callejo Gallego, J. (2010). Estudios Superiores En La Educación Penitenciaria Española: Un Análisis Empírico A Partir De Los Actores. Revista De Educación, 443-468.

Aysha Kerr, M. W. (2018). Prisoner Use Of Information And Communications Technology. Trends \& Issues In Crime And Criminal Justice.

Diez Palomar, J. (2009). La Enseñanza De Las Matemáticas A Personas Adultas Desde Un Enfoque Didáctico Basado En El Aprendizaje Dialógico. Enseñanza De Las Ciencias, 369380 .

Salamanca García, L. (2016). Intervención Educativa En Prisión: Memoria Y Deseo. Revista De Educación Social, 159-173.

Añaños , F. (2012). Violencias y exclusiones en el medio penitenciario. Enfoque socio-educativo y de la paz. Convergencia, 13-41. 
Hernández Jiménez, N. (2017). La Resocialización Como Fin De La Pena- Una Frustración Del Sistema Penitenciario $Y$ Carcelario Colombiano. Cuaderno Crh, 539-559.

C. Crabbe, M. J. (2016). Education For Offenders In Prison. Journal Of Pedagogic Development, 3-7.

Aishah, S., \& Zeema, S. (2017). The Ict Facilities, Skills, Usage, And The Problems Faced By The Students Of Higher Education. Modestum, 49874994.

Del Pozo Serrano, F. J., Jiménez Bautista, F., \& Turbi Pinazo, Á. M. (2013). El Tratamiento Con Mujeres: Actuación Socioeducativa $Y$ Sociolaboral En Prisiones. Pedagogía Social, 57-72.

Ayuso, A. (2001). La Intervención Socioeducativa En El Tratamiento Penitenciario. Pedagogía Social, 73-99.

Brasil, U. (2008). Proyecto Educando Para La Libertad Y Su Interfase Con EI Pronasci. En U. Brasil, Educación En Prisiones En Latinoamérica (Págs. 17-19). Brasilia.

Castillo Algarra, J., \& Ruiz García, M. (2007). Un Reto Educativo En EI Siglo Xxi: La Educación De Delincuentes Dentro Del Ámbito Penitenciario. Una Perspectiva De Géneros. Revista De Educación, 301-314. 